ARTICLE

\title{
Long non-coding RNA-mediated transcriptional interference of a permease gene confers drug tolerance in fission yeast
}

\author{
Ryan Ard ${ }^{1}$, Pin Tong $^{1} \&$ Robin C. Allshire ${ }^{1}$
}

Most long non-coding RNAs (IncRNAs) encoded by eukaryotic genomes remain uncharacterized. Here we focus on a set of intergenic IncRNAs in fission yeast. Deleting one of these IncRNAs exhibited a clear phenotype: drug sensitivity. Detailed analyses of the affected locus revealed that transcription of the nc-tgp1 IncRNA regulates drug tolerance by repressing the adjacent phosphate-responsive permease gene transporter for glycerophosphodiester $1\left(\operatorname{tgp} 1^{+}\right)$. We demonstrate that the act of transcribing $n c$-tgp1 over the $\operatorname{tgp} 1^{+}$promoter increases nucleosome density, prevents transcription factor access and thus represses $\operatorname{tgp} 1^{+}$without the need for RNA interference or heterochromatin components. We therefore conclude that $\operatorname{tgp} 1^{+}$is regulated by transcriptional interference. Accordingly, decreased nc-tgp1 transcription permits $\operatorname{tgp} 1^{+}$expression upon phosphate starvation. Furthermore, nc-tgp1 loss induces $\operatorname{tgp} 1^{+}$even in repressive conditions. Notably, drug sensitivity results directly from tgp $1^{+}$expression in the absence of the nc-tgp1 RNA. Thus, transcription of an IncRNA governs drug tolerance in fission yeast.

\footnotetext{
${ }^{1}$ Wellcome Trust Centre for Cell Biology and Institute of Cell Biology, School of Biological Sciences, The University of Edinburgh, Max Born Crescent, Edinburgh EH9 3BF, Scotland, UK. Correspondence and requests for materials should be addressed to R.C.A. (email: robin.allshire@ed.ac.uk).
} 
E ukaryotic genomes are pervasively transcribed. Frequently this transcription generates long non-coding RNAs (lncRNAs), which may be transcribed antisense to protein-coding genes, from within introns, or from intergenic regions of the genome. RNA polymerase II (RNAPII) is responsible for generating both messenger RNAs (mRNAs) and lncRNAs ${ }^{1}$. As with mRNAs, many lncRNAs are processed (that is, capped, spliced, polyadenylated), however, in contrast to protein-coding mRNAs, lncRNAs are predominantly nuclear and many are rapidly degraded by the exosome $e^{2}$, the major cellular $3^{\prime} \rightarrow 5^{\prime}$ RNA degradation machinery ${ }^{3}$. Consequently, the majority of lncRNAs exhibit low steady-state levels compared with mRNAs. This instability coupled with their general lack of primary sequence conservation has lead to the suggestion that many lncRNAs might simply result from spurious, inconsequential 'transcriptional noise ${ }^{4}$. Nonetheless, accumulating evidence indicates that an increasing number of lncRNAs act to regulate gene expression ${ }^{2,5}$.

The mere act of IncRNA transcription, including accompanying chromatin modifications and resulting changes in nucleosome density $^{6}$, can have a profound impact on neighbouring gene expression. In the simplest scenario, lncRNA expression can provide an environment that is either suitable or unsuitable for transcription factor binding. For example, cascading lncRNA transcription upstream of the fission yeast Schizosaccharomyces pombe $f b p 1^{+}$gene is required to induce $f p b 1^{+}$expression following glucose starvation ${ }^{7}$. In addition, in a process termed 'transcriptional interference', serine-mediated repression of the budding yeast Saccharomyces cerevisiae SER3 gene is brought about by lncRNA transcription into the gene promoter, which increases nucleosome density and prevents transcription factor access $^{8-10}$. These examples illustrate the positive and negative influence that lncRNA transcription can exert on gene regulation in response to environmental changes.

lncRNAs can also be processed into smaller regulatory RNAs (for example, short interfering RNA) ${ }^{11}$. In $S$. pombe, lncRNAs transcribed from centromeric outer repeats are processed by Dicer (Dcr1) into short interfering RNAs, which target the Clr4 H3K9 methyltransferase via Agol (within the RNA-induced transcriptional silencing complex) to establish repressive heterochromatin through the methylation of lysine 9 on histone H3 (refs 12-15). In addition, lncRNAs may directly associate with and recruit factors that alter chromatin status, in cis or in trans, silencing genes or behaving as enhancers ${ }^{16,17}$. For example, lncRNAs aid the response of $S$. cerevisiae cells to specific changes in nutrient availability by recruiting chromatin-modifying complexes (for example, histone deacetylases) to dynamically regulate several genes ${ }^{18-20}$. Related mechanisms have since been reported in multicellular eukaryotes ${ }^{21,22}$. Recent analyses also suggest that patches of transient heterochromatin can form under particular conditions at specific euchromatic loci in S. pombe $23-25$. This mechanism involves the RNA-binding protein Mmil, which recruits the RNA-surveillance machinery to specific determinant of selective removal (DSR) motifs in target transcripts, leading to their exosome-mediated degradation $^{26}$. Mmil and its associated factor Red1 are reported to also recruit chromatin-modifying activities via nascent mRNA and lncRNA targets to deposit H3K9 methylation (H3K9me2) at these locations $23,25,27,28$. It is therefore evident that IncRNAs employ a variety of mechanisms to regulate gene expression.

Despite rapid advances in lncRNA identification, only a small number have been characterized in detail. A clear challenge in assigning function has been a lack of lncRNA sequence conservation between even the most closely related species ${ }^{29}$. However, the order of genes flanking the transcription units that encode lncRNAs can be preserved through evolution ${ }^{30}$ (that is, synteny) and provides another criterion by which we can identify potential functionally conserved lncRNAs whose primary sequences might have diverged too much so as not to retain detectable homology.

Only a few of the $\sim 500$ annotated intergenic lncRNAs in $S$. pombe are conserved at the sequence level in three divergent Schizosaccharomyces species, although many retain synteny with flanking genes in at least one other species ${ }^{31}$. We identified eight discrete intergenic lncRNAs that exhibit synteny in at least three of the four Schizosaccharomyces species. Deletion of one of these loci (SPNCRNA.1343 or ncRNA.1343 for short) exhibited a drugsensitivity phenotype. We demonstrate that $n c R N A .1343$ encodes a bidirectional lncRNA promoter and that its deletion causes loss of expression of the divergent unstable transcript $n c$-tgp1. Our analyses reveal that $n c$-tgp1 is targeted for Mmil-directed exosome degradation and is required to repress a downstream phosphate-responsive gene (SPBC1271.09 designated transporter for glycerophosphodiester $\left.1\left(\operatorname{tgp} 1^{+}\right)\right)$. However, rather than involving transient heterochromatin formation as a result of targeted RNA degradation, the regulation of $\operatorname{tgp} 1^{+}$by the $n c$ - $\operatorname{tg} p 1$ RNA appears to be mediated by transcriptional interference. Most importantly, tolerance of $S$. pombe to a broad spectrum of compounds relies on the regulation of $\operatorname{tgp} 1^{+}$by $n c$ - $\operatorname{tgp} 1$.

\section{Results}

Deletion of SPNCRNA.1343 causes drug hypersensitivity. The $S$. pombe genome is predicted to encode $\sim 500$ intergenic lncRNAs ${ }^{32}$. Although few of these lncRNAs exhibit detectable sequence conservation, $\sim 100$ are conserved in synteny with putative lncRNA orthologues in at least one of the three other known Schizosaccharomyces species ${ }^{31}$. For example, the functionally characterized telomerase RNA (ter $1^{+}$/SPNCRNA.214) is syntenic despite its lack of sequence conservation (see Supplementary Fig. 1a).

To identify other potential functionally conserved lncRNAs, we selected eight lncRNAs, including ter ${ }^{+}$as a control, where surrounding gene order is retained in $S$. pombe and at least two other Schizosaccharomyces species. Each lncRNA gene was deleted by replacement with a loxP-flanked rra $^{+}$marker (Supplementary Fig. 1b). Apart from ter1 1 , the selected lncRNAs were not essential for normal cell growth (Supplementary Figs 1c and 2). However, since many characterized lncRNAs regulate gene expression in response to environmental changes and stress $^{33}$, we tested the growth of these lncRNA deletion strains in response to the following stresses: temperature, the microtubule destabilizing drug thiabendazole (TBZ), DNA synthesis inhibitor hydroxyurea ( $\mathrm{HU})$, ultraviolet-induced DNA damage, $\mathrm{H}_{2} \mathrm{O}_{2}$ induced oxidative stress and caffeine, an inhibitor of cyclic AMP phosphodiesterase. Cells lacking SPNCRNA.1343 (ncRNA.1343 for short) displayed a phenotype: hypersensitivity to TBZ, HU and caffeine but not to temperature extremities, ultravioletirradiation or oxidative stress (Supplementary Fig. 1c and Supplementary Fig. 2).

Drug sensitivity of 13434 cells is caused by $\operatorname{tgp} 1^{+}$induction. lncRNAs can act in cis to regulate the expression of nearby genes $^{2}$. To determine the cause of drug sensitivity in $1343 \Delta$ cells we examined the expression of genes flanking the locus by real-time quantitative reverse transcriptase-PCR (RT-qPCR) in wild-type cells, cells with $n c R N A .1343$ replaced by loxP-flanked ura $^{+}$marker $\left(13434::\right.$ ura $\left.^{+}\right)$and cells with the ura $^{+}$marker subsequently removed (13434; Fig. 1a). SPBC1271.09 transcript levels increased $>50$-fold in both 13434::ura4 ${ }^{+}$and 13434 cells (Fig. 1b), while the expression of other neighbouring genes was unaltered. SPBC1271.09 encodes a conserved 


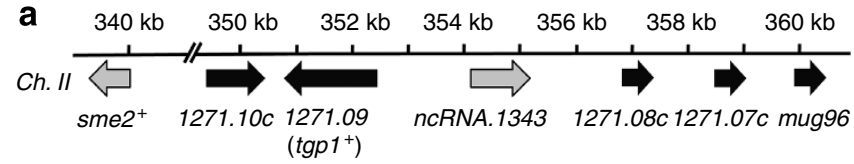

b
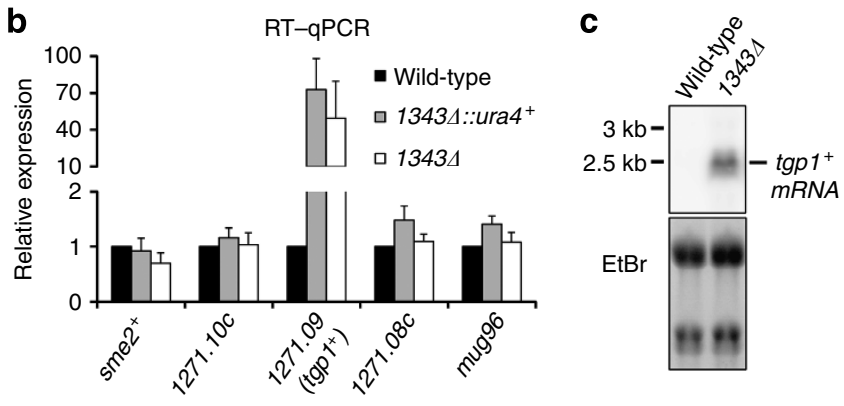

d

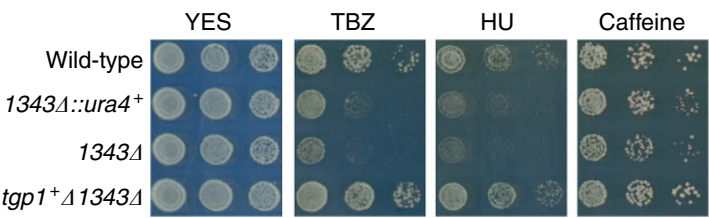

Figure 1 | Drug sensitivity following ncRNA.1343 deletion is due to increased $\mathbf{t g p} \mathbf{1}^{+}$expression. (a) Schematic representation of genes flanking ncRNA.1343. (b) RT-qPCR experiments measured transcript levels for nearby genes in wild-type cells and following replacement of ncRNA.1343 with ura4 ${ }^{+}\left(13434::\right.$ ura4 $\left.^{+}\right)$or deletion (13434). Error bars represent s.e.m. resulting from at least three independent replicates. (c) Northern analysis of tgp $1^{+}$transcript levels in wild-type and 13434 cells grown in the presence of phosphate. (d) Serial dilutions of wild-type, 13434::ura ${ }^{+}, 1343 \Delta$ and tgp1 13434 double mutant spotted on nonselective YES medium or in the presence of TBZ $\left(20 \mu \mathrm{gl}^{-1}\right), \mathrm{HU}(10 \mathrm{mM})$ or caffeine $(15 \mathrm{mM})$, respectively.

glycerophosphodiester membrane transporter (designated as $\operatorname{tg} 1^{+}$) orthologous to the $S$. cerevisiae permease GIT1. As with S. cerevisiae GIT1, the $\operatorname{tgp} 1^{+}$gene is repressed when cells are grown in the presence of phosphate and induced upon phosphate starvation ${ }^{34,35}$. Northern analysis confirmed that $\operatorname{tg} p 1^{+}$was indeed highly expressed in 13434 cells but not wild-type cells, both grown in the presence of phosphate (repressed condition; Fig. 1c).

To determine whether the drug sensitivity of 13434 cells is a direct result of increased $\operatorname{tgp} 1^{+}$expression, the $\operatorname{tg} p 1^{+}$gene was deleted from 13434 cells (tgp1 13434). This manipulation restored TBZ, HU and caffeine tolerance to levels comparable with wild-type cells (Fig. 1d). We conclude that increased $\operatorname{tgp} 1^{+}$ expression is directly responsible for the drug-sensitivity phenotype of cells lacking $n c R N A .1343$.

Bidirectional lncRNA promoter upstream of $\operatorname{tgp} 1^{+}$. Previous RNA-seq analysis indicates that an IncRNA is transcribed in the sense orientation upstream of $\operatorname{tgp} 1^{+}$(refs 27,31). We identified two divergent transcriptional start sites arising within $n c R N A .1343$ : one $\operatorname{lncRNA}$ transcribed towards the $\operatorname{tgp} 1^{+}$gene $(n c-\operatorname{tg} p 1)$ and the other in the opposite orientation (nc-1343; Fig. 2a; Supplementary Fig. 3). lac $Z$ reporter assays demonstrate that the bidirectional promoter drives greater levels of transcription in the $n c$-tgp1 direction (Supplementary Fig. 3). This finding is consistent with Rpb1 Chromatin Immunoprecipitation (ChIP) analysis showing that RNAPII is enriched over the $n c$-tgp 1 transcription unit, while much lower RNAPII levels are detected on $n c-1343$ (Fig. $2 \mathrm{~b}$ ).
We next examined the regulation of the $n c-1343$ and $n c$-tgp 1 transcripts produced from this bidirectional promoter. A $\sim 0.9 \mathrm{~kb}$ transcript for $n c-1343$ was readily detected in wild-type cells. The size and levels of the $n c-1343$ transcript increased in exosome defective (rrp64) cells, but not cells lacking Mmil or Red1 (Fig. 2c,d; Supplementary Fig. 4). The IncRNA corresponding to $n c$-tgp 1 was previously detected in $r r p 64$ and red14 cells ${ }^{27}$. We identified a consensus DSR motif for Mmil binding at position $+820 \mathrm{nt}$ within the $n c$-tgpl transcript and RNA IP (RIP) experiments confirmed a direct interaction between Mmil and the nc-tgp1 RNA (Supplementary Fig. 5). Northern analysis identified that an $\sim 1.9 \mathrm{~kb} n c$-tgpl RNA accumulates in $\operatorname{rrp} 6 \Delta$, mmild and red14, but not in wild-type cells (Fig. 2e,f; Supplementary Fig. 4). Interestingly, a recent study found that the repressive lncRNA transcribed upstream of the phosphate-responsive $\mathrm{phol}^{+}$gene in S. pombe also contains a DSR motif and is targeted by Mmil for exosome-mediated degradation $^{28}$, indicating that a similar regulatory mechanism might control expression of $\operatorname{tgp} 1^{+}$and $p h o 1^{+}$. In sum, both $n c-1343$ and $n c$-tgp 1 transcripts are processed by the exosome, but only $n c-\operatorname{tg} 1$ is regulated by Mmil-mediated recruitment of the nuclear exosome.

A moderate increase in $\operatorname{tgp} 1^{+}$transcript levels has previously been reported in cells lacking Mmil (ref. 23). In agreement with this, we detected a similar increase (approximated four-fold) in $\operatorname{tgp} 1^{+}$transcript levels in mmild or exosome ( $r p 64$ or dis3-54) mutant cells by RT-qPCR, however, this increase is significantly less than the $>50$-fold upregulation of $\operatorname{tgp} 1^{+}$observed in 13434 cells (Fig. 2g,h; Supplementary Fig. 4). Moreover, we failed to detect the $\operatorname{tgp} 1^{+}$transcript in rrp64 or mmila cells by Northern analysis, indicating that $\operatorname{tgp} 1^{+}$is not induced in the absence of these factors. Thus, Mmil-mediated exosome degradation is not the predominant mechanism involved in $\operatorname{tgp} 1^{+}$regulation.

$\operatorname{tgp} 1^{+}$is repressed by the $\boldsymbol{n c - t g p 1} \operatorname{lncRNA}$. The presence of the unstable $n c$-tgp $1 \mathrm{RNA}$ upstream of $\operatorname{tgp} 1^{+}$suggests that either $n c$ $\operatorname{tgp} 1, n c-1343$ or both regulate $\operatorname{tg} p 1^{+}$expression. To test the involvement of these $\operatorname{lncRNAs}$ in $\operatorname{tgp} 1^{+}$regulation, a series of strategic genetic manipulations were performed (Fig. 3a). Truncations of $n c-1343$ (that is, $A \Delta$ and $B \Delta$ ) that retain its $5^{\prime}$ end did not result in the drug-sensitivity phenotype presented by 13434 cells (Fig. 3b) and, similarly, did not induce tgp $1^{+}$expression (Fig. 3c). This indicates that full-length $n c-1343$ is not required for $\operatorname{tgp} 1^{+}$repression. We next tested if $n c$-tgp 1 is involved in repressing $\operatorname{tgp} 1^{+}$. Our analyses show that transcription of $n c-\operatorname{tg} p 1$ starts within the encoded ncRNA.1343 transcription unit (Supplementary Fig. 3). Thus, deletion of the entire locus (13434) removes the $n c$-tgp 1 promoter, and the $5^{\prime}$ end of its transcript, resulting in the observed loss of $n c$-tgp 1 expression (Figs $2 \mathrm{f}$ and $3 c$ ). The $A \Delta$ and $B \Delta$ truncations of $n c-1343$, which retain the $n c$-tgp 1 promoter, do not affect $n c$-tgp 1 transcription or relieve repression of $\operatorname{tg} \mathrm{P}^{+}$. In contrast, interruption of the $n c-\operatorname{tg} p 1$ transcription unit by insertion of the $\mathrm{ura}^{+}$marker gene $\left(n c\right.$-tgp 1:ura $\left.4^{+}\right)$prevented $n c$-tgp 1 transcription, induced tgp $1^{+}$ expression to levels observed in $1343 \Delta$ levels and increased sensitivity of these cells to TBZ, HU and caffeine (Fig. 3b,c). These analyses demonstrate that it is $n c$ - $\operatorname{tg} 1$, not $n c-1343$, that is critical for repressing $\operatorname{tgp} 1^{+}$in the presence of phosphate.

Phosphate starvation induces $\operatorname{tgp} 1^{+}$by repressing $n c-\operatorname{tg} p 1$. Upon phosphate starvation of fission yeast, several genes involved in the phosphate response are induced (including $\operatorname{tgp} 1^{+}$and $\left.p h 1^{+}\right)$(ref. 35). To determine how the transcription of $n c$-tgp 1 is altered in response to phosphate and how it might influence $\operatorname{tgp} 1^{+}$expression we assessed expression in phosphate-rich 

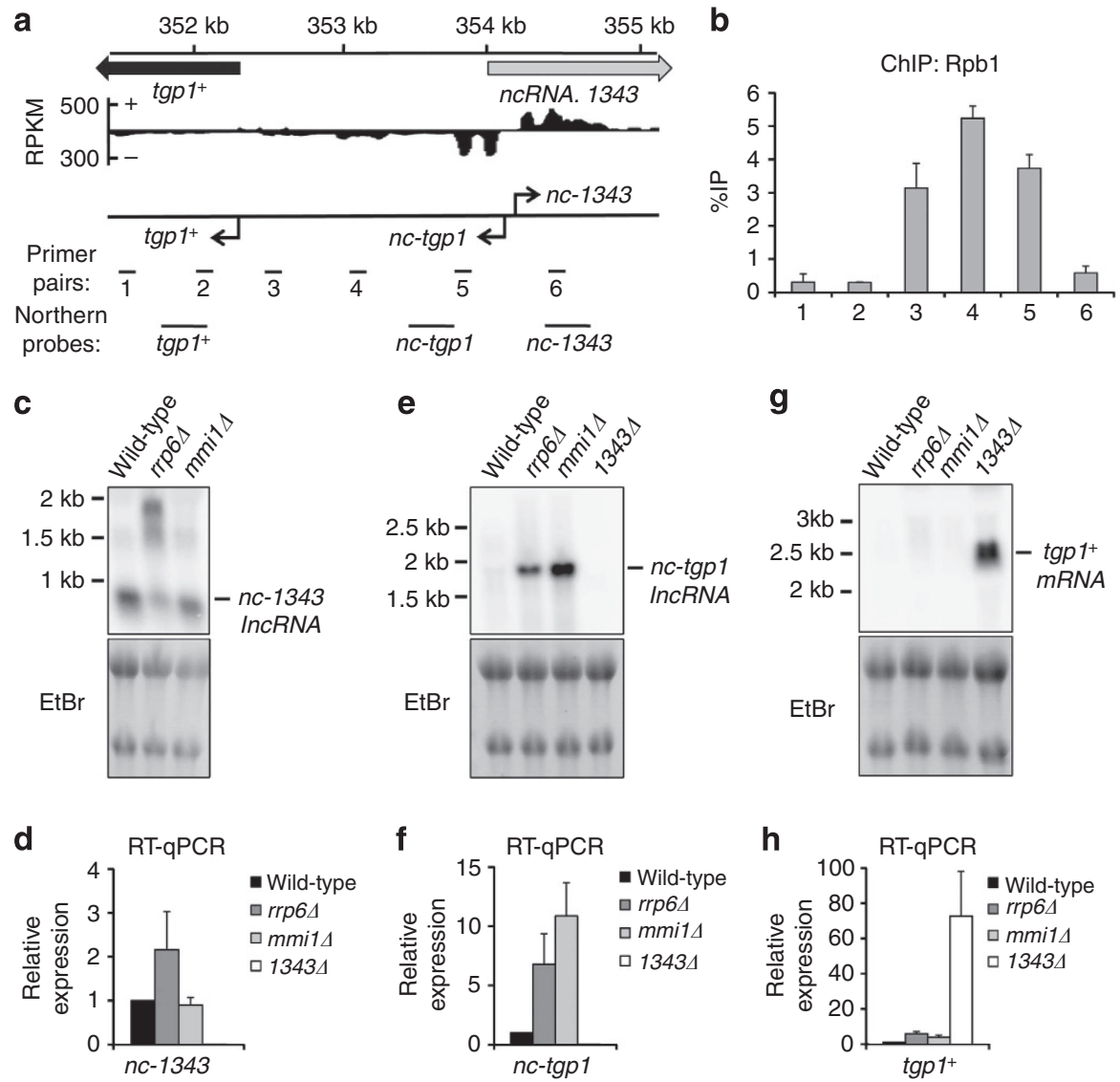

Figure 2 | Two distinct IncRNAs are transcribed from a bidirectional promoter upstream of $\boldsymbol{t g p}^{+}$. (a) Previously published strand-specific RNA-Seq analysis (Rhind et al., ${ }^{31}$ ) upstream of SPBC1271.09/tgp $1^{+}$, represented as reads per kilobase per million (RPKM). Location of qPCR primer pairs and probes for Northern analysis are shown below. (b) Rbp1 ChIP-qPCR experiments performed in wild-type cells. (c,e,g) Northern analysis of $n c-1343, n c-t g p 1$ and $\operatorname{tgp} 1^{+}$transcript levels in wild-type, rrp64, mmi14 and 13434, respectively. (d,f,h) RT-qPCR experiments measured nc-1343, nc-tgp1 and tgp $1^{+}$ transcript levels in wild-type, rrp64, mmi14 and 13434, respectively. Error bars represent s.e.m. resulting from at least three independent replicates.

$\left(+\mathrm{PO}_{4}\right)$ and phosphate-deprived $\left(-\mathrm{PO}_{4}\right)$ conditions. As expected, the levels of $\operatorname{tgp} 1^{+}$and the $p h o 1^{+}$control increased upon phosphate starvation (Fig. 4a,b). In contrast, the levels of both $n c-\operatorname{tgp} 1$ and $n c-1343$ RNAs decreased significantly in the absence of phosphate (Fig. 4a; Supplementary Fig. 6). The observed reduction in $n c$-tgp1 levels is consistent with a situation whereby loss or reduction of $n c$-tgp 1 transcription permits $\operatorname{tgp} 1^{+}$ induction. In agreement with this, significantly less RNAPII associates with the $n c$-tgp1 transcription unit in both phosphatestarved wild-type cells and phosphate-replete 13434 cells, which do not transcribe $n c$ - $\operatorname{tg} p 1$ (Fig. 4c). Therefore, preventing $n c-\operatorname{tg} p 1$ transcription, even in phosphate-rich medium, recapitulates the changes in RNAPII occupancy that normally accompany $\operatorname{tgp} 1^{+}$ induction upon phosphate deprivation.

RNAi-directed heterochromatin does not repress $\operatorname{tgp} 1^{+}$. Cells with defective exosome function (rrp64) accumulate non-coding RNAs, some of which have been reported to attract Mmildependent RNA elimination factors, along with RNA interference (RNAi) components and the Clr4 H3K9 methyltransferase, leading to the formation of transiently regulated HOODs (heterochromatin domains) $)^{25}$. The regions containing the $\operatorname{tgp} 1^{+}$and pho1 ${ }^{+}$genes are included in HOOD-17 and HOOD-24, respectively, and both form a region of Mmil-directed transient heterochromatin in rrp64 cells ${ }^{24,27}$. The $n c$-tgp1 transcript is clearly regulated by Mmil-directed exosome degradation
(Fig. 2e,f), however, we do not detect methylated $\mathrm{H} 3 \mathrm{~K} 9$ (H3K9me2) over the $\operatorname{tgp} 1^{+}, n c$-tgp1 or $n c-1343$ genes within HOOD-17 in wild-type cells (Fig. 5a). Likewise, only very low levels of $\mathrm{H} 3 \mathrm{~K} 9 \mathrm{me} 2$, slightly above background in cells lacking the H3K9 methyltransferase (clr44), could be detected on the pho1 ${ }^{+}$ gene and the upstream Mmil-targeted lncRNA (nc-pho1) within HOOD-24. Moreover, this low level of H3K9me2 did not drop appreciably upon induction of $\operatorname{tgp} 1^{+}$and $p h o 1^{+}\left(-\mathrm{PO}_{4}\right.$; Fig. 5a). Equivalent background levels of $\mathrm{H} 3 \mathrm{~K} 9 \mathrm{me} 2$ were detectable on another Mmil-targeted lncRNA gene $\left(s m e 2^{+}\right.$) and the highly expressed actin gene $\left(a c t 1^{+}\right)$. In contrast, H3K9me2 was $\sim 100$-fold enriched over the centromeric outer repeats $(d g)$ in wild-type cells, but reduced to background in clr4A cells, indicating that $\mathrm{H} 3 \mathrm{~K} 9$-methylated chromatin had been efficiently immunoprecipitated. In addition, the transcript levels of $\operatorname{tg} p 1^{+}, n c-\operatorname{tg} p 1, n c-1343, p h o 1^{+}$and $n c$ - $p h o 1$ were unaffected by loss of RNAi (for example, ago1A or dcr14) or heterochromatin components (for example, clr44 or swi64) (Fig. 5b; Supplementary Fig. 7a). Nor were the kinetics of $\operatorname{tg} p 1^{+}$ or $\mathrm{phol}^{+}$induction following phosphate starvation altered in cells lacking heterochromatin (Supplementary Fig. 7b,c). In contrast, $n c-\operatorname{tgp} 1, n c-p h o 1$ and $s m e 2^{+}$RNA levels were clearly elevated in cells lacking Mmil-mediated exosome degradation (mmi14 and rrp64). Thus, although H3K9me2 accumulates at particular regions in $\operatorname{rrp6} 6$ cells (for example, HOOD-17: $\operatorname{tgp} 1^{+}$ and HOOD-24: $\left.p h o 1^{+}\right)$, we conclude that RNAi and heterochromatin play no appreciable role in regulating these 
a

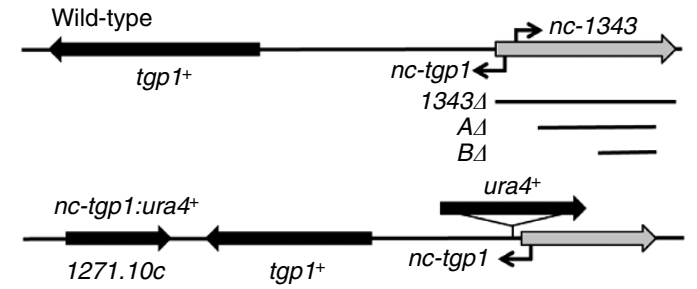

b
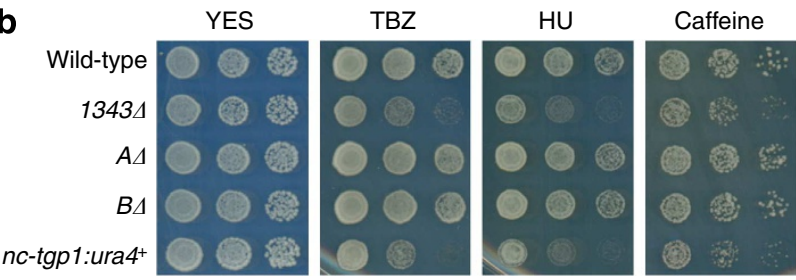

C

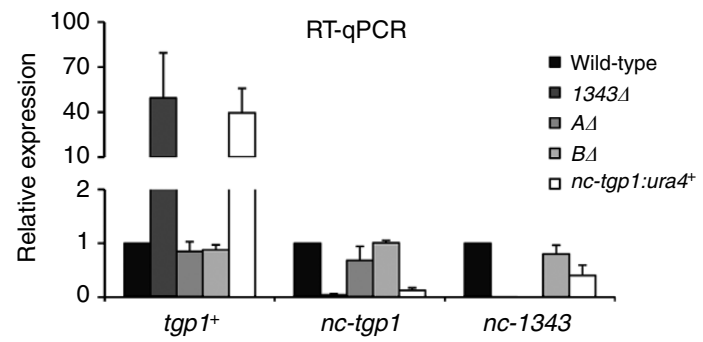

Figure 3 | nc-tgp1, not nc-1343, represses tgp1 ${ }^{+}$to confer drug tolerance. (a) Schematic diagram indicating strategic manipulations of IncRNAs upstream of $\operatorname{tgp} 1^{+}$including 13434, shorter deletions of ncRNA.1343 ( $A \Delta$ and $B \Delta$ ) and ura $4+$ integration within the nc-tgp 1 IncRNA locus (nc-tgp1:ura4 ${ }^{+}$) in wild-type background. (b) Serial dilutions of wild-type, 13434, A4, BA and nc-tgp1:ura ${ }^{+}$were spotted on non-selective YES medium or in the presence of TBZ $\left(20 \mu \mathrm{g} \mathrm{ml}^{-1}\right), \mathrm{HU}(10 \mathrm{mM})$ or caffeine $(15 \mathrm{mM})$, respectively. (c) RT-qPCR experiments measured tgp $1^{+}$, nc-tgp1 and nc-1343 transcript levels in wild-type, 13434, A4, BA and nc-tgp1:ura4 ${ }^{+}$ cells. Error bars represent s.e.m. resulting from three independent replicates.

genes under normal physiologically repressive conditions or during their induction.

nc-tgp1 prevents Pho7 transcription factor binding. The above analyses indicate that $n c$ - $\operatorname{tg} p 1$ is transcribed into the $\operatorname{tgp} 1^{+}$promoter and suggest that production of this upstream lncRNA represses $\operatorname{tgp} 1^{+}$expression. We therefore investigated if transcription of $n c$ - $\operatorname{tgp} 1$ interferes with the induction mechanism of $\operatorname{tgp} 1^{+}$in response to phosphate starvation. The Pho7 transcription factor has previously been shown to engage phosphateresponse gene promoters in phosphate-starved cells ${ }^{35,36}$. Our ChIP analyses confirmed that Pho7-green fluorescent protein (Pho7-GFP) accumulates on the $\mathrm{phol}^{+}$promoter in phosphatedepleted cells (Supplementary Fig. 8). In addition, Pho7-GFP levels accumulate over the region upstream of $\operatorname{tgp} 1^{+}$when activated (Fig. 6a). However, in cells unable to transcribe $n c$-tgp1 (13434), higher levels of Pho7-GFP associate with the region upstream of $\operatorname{tgp} 1^{+}$even in repressive conditions (that is, $+\mathrm{PO}_{4}$ ). We conclude that loss of $n c$-tgpl expression due to phosphate starvation or by preventing production of this lncRNA (for example, 13434) allows Pho7 binding and subsequent $\operatorname{tgp} 1^{+}$ induction.

Active RNAPII promoters display reduced nucleosome density ${ }^{37}$. IncRNA transcription over promoters can increase nucleosome density and prevent gene induction ${ }^{8,10,20}$. We a

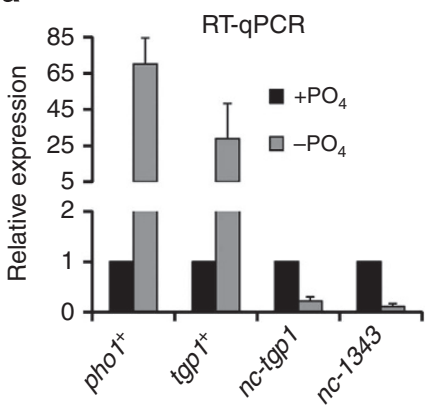

b

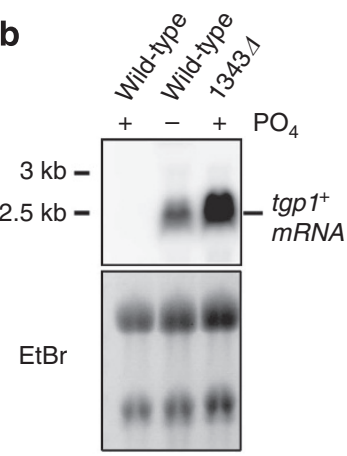

C
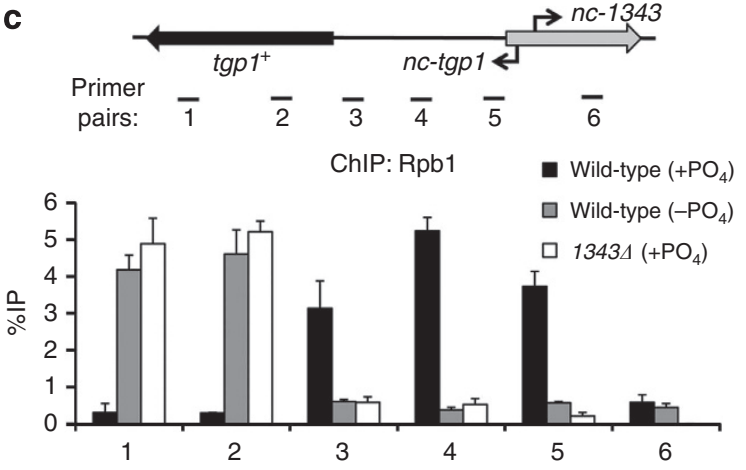

Figure 4 | Phosphate starvation induces tgp ${ }^{+}$and reduces IncRNA transcription. (a) RT-qPCR experiments measured tgp $1^{+}$, nc-tgp 1 and $n c-1343$ transcript levels in wild-type cells grown in phosphate-rich medium $\left(+\mathrm{PO}_{4}\right)$ or in the absence of phosphate $\left(-\mathrm{PO}_{4}\right) \cdot p h o 1^{+}$is a positive control for phosphate starvation. (b) Northern analysis of tgp $1^{+}$in wildtype cells grown in the presence or absence of phosphate and 13434 grown in the presence of phosphate. (c) Rbp1 ChIP-qPCR experiments performed in wild-type cells grown in the presence or absence of phosphate and 13434 grown in the presence of phosphate. Error bars represent s.e.m. resulting from three independent replicates.

found that histone $\mathrm{H} 3$ levels were greater over the $\operatorname{tgp} 1^{+}$gene and upstream region when it is repressed $\left(+\mathrm{PO}_{4}\right)$ compared with when it is expressed ( $-\mathrm{PO}_{4}$; Fig. $\left.6 \mathrm{~b}\right)$. In contrast, $\mathrm{H} 3$ levels over control loci $\left(a c t 1^{+}, s m e 2^{+}\right.$and $d g$ repeats) were unaffected by phosphate availability. Thus, upstream transcription appears to alter nucleosome density over the $\operatorname{tg} p 1^{+}$promoter and thereby occlude Pho7 binding. Likewise, a considerable drop in $\mathrm{H} 3$ levels was observed on the pho1 ${ }^{+}$gene and nc-phol lncRNA region upstream in phosphate-poor conditions, implying a similar mechanism may also operate to regulate the expression of pho $1^{+}$. We conclude that transcription of the upstream lncRNA inhibits expression of $\operatorname{tg} p 1^{+}$by a transcriptional interference mechanism that alters the chromatin landscape, preventing access to the key phosphate-responsive transcription factor Pho7.

To directly test if transcriptional interference of $\operatorname{tgp} 1^{+}$by $n c$ $\operatorname{tgp} 1$ is responsible for $\operatorname{tgp} 1^{+}$repression, we replaced the $n c$-tgp1 promoter with the strong, thiamine-regulated $n m t 1$ promoter (nmt1-nc-tgp1) (Fig. 7a). Transcription of $n c$-tgp1 from the nmt1 promoter is rendered unresponsive to phosphate. Instead, $n c$-tgp 1 is repressed or derepressed in the presence or absence of thiamine, respectively. When $n c$-tgp 1 was transcribed from the $n m t 1$ promoter, $\operatorname{tgp} 1^{+}$remained repressed regardless of phosphate availability (Fig. $7 \mathrm{~b}$ ). In contrast, repression of $n m t 1$ driven $n c-\operatorname{tg} p 1$ by thiamine resulted in the induction of $\operatorname{tgp} 1^{+}$ expression in phosphate-rich media and consequently caused drug sensitivity (Fig. $7 \mathrm{~b}, \mathrm{c}$ ). In addition, $\mathrm{H} 3$ levels over the region upstream of $\operatorname{tgp} 1^{+}$were high when $n c$-tgp1 was transcribed and 

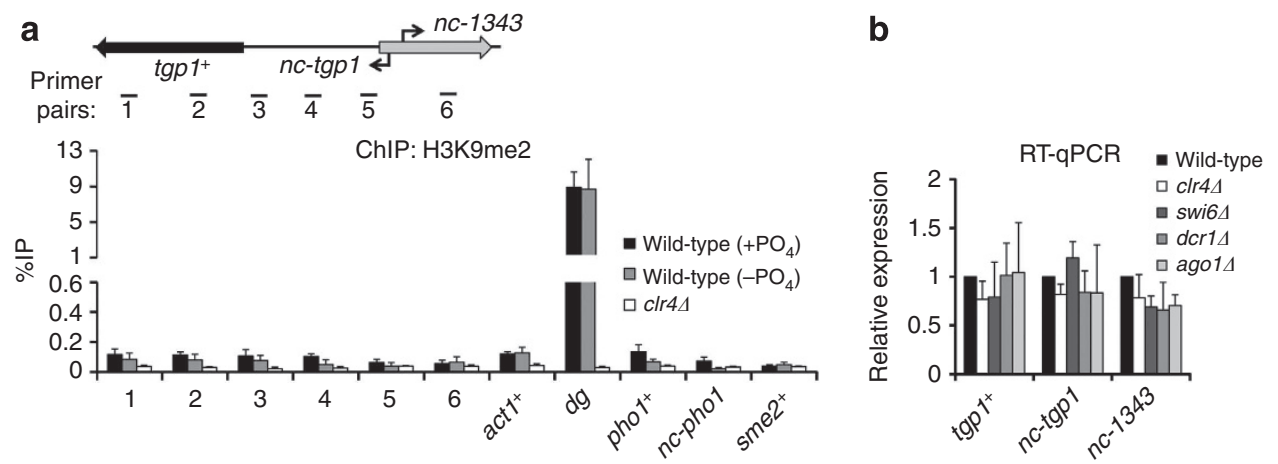

Figure 5 | tgp1 $^{+}$is not regulated by RNAi/heterochromatin. (a) H3K9me2 ChIP-qPCR experiments performed in the presence or absence of phosphate. clr $4 \Delta$ was used as a negative control. The euchromatic actin gene $\left(a c t 1^{+}\right)$and centromeric $d g$ repeats $(d g)$ are positive and negative controls for heterochromatin. pho1 ${ }^{+}$is a phosphate-regulated gene repressed by nc-pho1, a IncRNA target of Mmi1. sme2 ${ }^{+}$is another IncRNA target of Mmi1. $\mathrm{H} 3 \mathrm{~K} 9 \mathrm{me} 2$ to bulk $\mathrm{H} 3$ ratio has not been presented due to background methyl H3K9 levels detected at these loci. (b) RT-qPCR experiments measured $\operatorname{tgp} 1^{+}, n c-t g p 1$ and nc-1343 transcript levels in wild-type cells and cells lacking factors involved in heterochromatin formation and stability, respectively. Error bars represent s.e.m. resulting from at least three independent replicates.

a

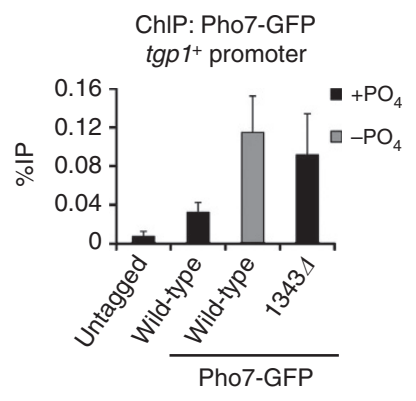

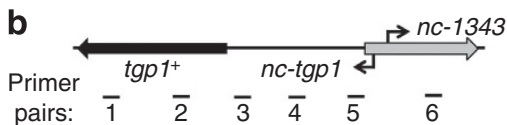

ChIP: Histone $\mathrm{H} 3$

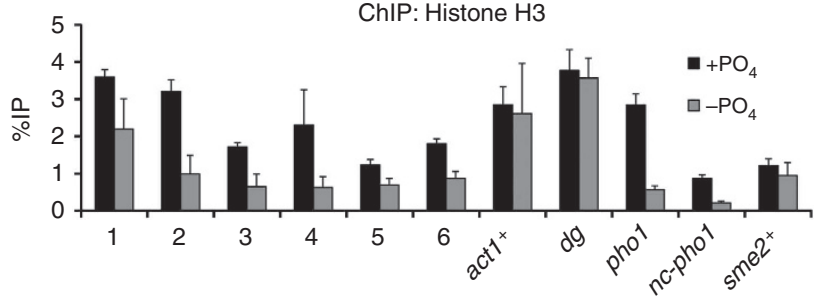

Figure 6 | nc-tgp1 transcription prevents stable Pho7 binding and increases nucleosome density upstream of tgp1 ${ }^{+}$. (a) GFP ChIP-qPCR experiments were performed in the presence or absence of phosphate in cells with C-terminally GFP-tagged Pho7. An untagged strain was used as a negative control. Primer pair \#3 was used to detect Pho7 binding at the tgp1 ${ }^{+}$promoter. (b) Nucleosome density was measured by histone H3 ChIP-qPCR experiments in wild-type cells grown in the presence or absence of phosphate. Error bars represent s.e.m. resulting from three independent replicates.

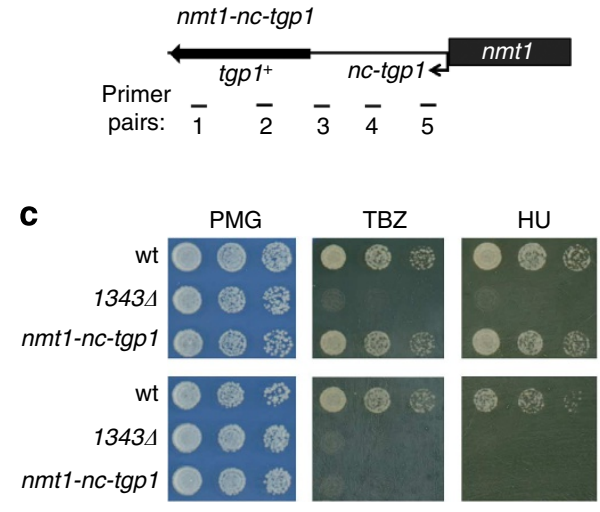

C

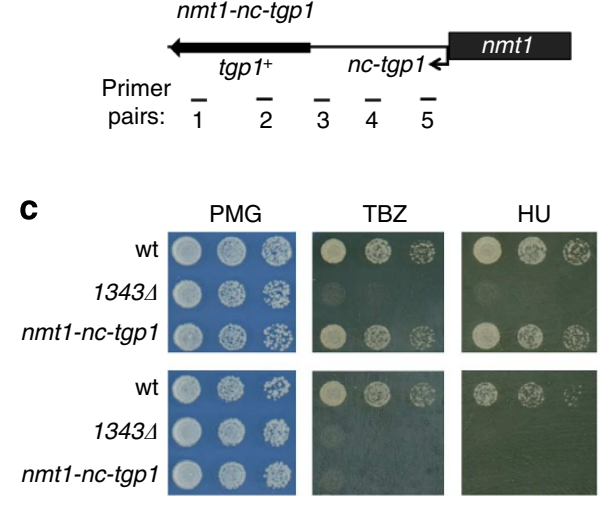

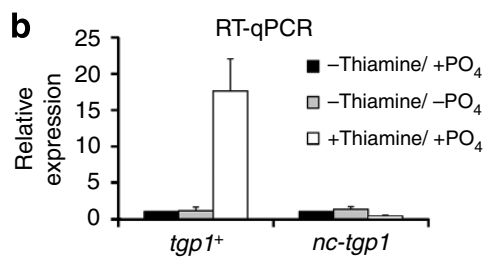

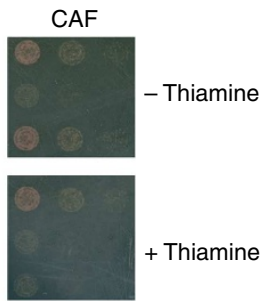

Figure 7 | nmt1 controlled nc-tgp1 alters drug tolerance in response to thiamine. (a) Schematic diagram of nc-tgp1 under the control of the strong, thiamine-repressible nmt1 promoter. (b) RT-qPCR experiments measured tgp $1^{+}$and $n c$-tgp 1 levels in response to thiamine and phosphate availability using nmt1-nc-tgp1 cells. (c) Serial dilutions of wild-type, 13434 and nmt1-nc-tgp1 cells were spotted on non-selective PMG medium or in the presence of TBZ, HU or caffeine, respectively, with or without thiamine as indicated. (d) H3 ChIP-qPCR experiments in nmt1-nc-tgp1 cells grown in the presence or absence of thiamine. Error bars represent s.e.m. resulting from three independent replicates.

reduced when $n c$ - $\operatorname{tg} p 1$ was repressed by thiamine (Fig. $7 \mathrm{~d}$ ). Lastly, exogenous expression of full-length $n c$-tgp 1 from a plasmid failed to repress $\operatorname{tgp} 1^{+}$, ruling out the possibility that $n c$-tgp 1 operates in trans (Supplementary Fig. 9). Collectively, these findings confirm that it is the transcription of $n c$ - $\operatorname{tg} p 1$ over the $\operatorname{tgp} 1^{+}$ promoter that alters nucleosome density to regulate $\operatorname{tg} p 1^{+}$ induction (see Fig. 8) and, as a consequence, drug tolerance of fission yeast cells. 

$+\mathrm{PO}_{4}$
(Repressed)
$-\mathrm{PO}_{4}$ or
Preventing $n c$-tgp 1 transcription (induced)

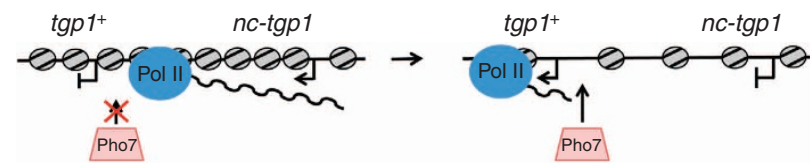

Figure 8 | Model for transcriptional interference at $\operatorname{tgp} 1^{+}$. The presence of phosphate induces transcription of an unstable IncRNA (nc-tgp1). IncRNA transcription increases nucleosome density, occludes Pho7 transcription factor binding and thus represses tgp $1^{+}$expression. nc-tgp 1 transcription is reduced following phosphate starvation, decreasing nucleosome density, allowing Pho7 to stably engage the $\operatorname{tgp} 1^{+}$promoter and induce $\operatorname{tgp} 1^{+}$ expression.

\section{Discussion}

An increasing number of lncRNAs have been shown to tightly regulate eukaryotic gene expression following intra-/extra-cellular environment changes that require rapid, integrated responses at the level of transcription ${ }^{2}$. In $S$. pombe, for example, the balance of antisense lncRNAs and sense transcription controls various stress-response pathways ${ }^{33,38}$. However, little is known about the majority of $S$. pombe intergenic lncRNAs. Here we selected and deleted eight stable, discrete lncRNAs in $S$. pombe that show conserved synteny in at least two of the three other known Schizosaccharomyces species. Excluding the ter $1^{+}$control, only deletion of $n c R N A .1343$ exhibited a definitive phenotype: sensitivity to various compounds due to induction of a nearby phosphate-responsive permease gene $\left(\operatorname{tgp} 1^{+}\right)$. Closer inspection revealed that the $n c R N A .1343$ promoter is bidirectional. Furthermore, transcription from this bidirectional promoter favours the production of a previously unannotated and unstable lncRNA (nc-tgp1) towards the $\operatorname{tg} p 1^{+}$gene under repressive conditions.

Recent studies in fission yeast have implicated lncRNAs in directing repression of specific genes by a mechanism involving transient RNAi-dependent heterochromatin formation ${ }^{27}$. For example, the Mmil-targeted IncRNA upstream of pho ${ }^{+}$has recently been reported to recruit RNAi-directed heterochromatin to repress $\mathrm{phol}^{+}$in response to phosphate availability ${ }^{28}$. However, these findings differ from genome-wide H3K9me2 mapping which show that $\operatorname{tgp} 1^{+}$and $p h o 1^{+}$, both of which are regulated by upstream lncRNAs that are targeted for exosomemediated degradation by Mmil (Fig. 2; ref 28), only accumulate RNAi-directed $\mathrm{H} 3 \mathrm{~K} 9 \mathrm{me} 2$ in mutants with defective RNA processing/degradation (for example, rrp64) and not in wildtype cells grown under repressive phosphate-rich conditions ${ }^{24}$. The significance of rrp64-dependent heterochromatin at the $\operatorname{tgp} 1^{+}$and $p h o 1^{+}$genes is therefore unclear. Cells lacking Rrp6 accumulate aberrant RNAs and exhibit disrupted heterochromatin globally, including significantly decreased H3K9me2 over centromeric repeats ${ }^{39}$. Therefore caution must be exercised when interpreting the analyses of mutants with such severe defects in RNA processing/degradation. Importantly, we do not detect significant levels of $\mathrm{H} 3 \mathrm{~K} 9 \mathrm{me} 2$ enrichment on the $\operatorname{tgp} 1^{+}$and $\mathrm{phol}^{+}$promoters/genes in wild-type cells under repressive (phosphate-rich) conditions. We cannot exclude the possibility that distinct assay conditions in a previous report allowed detection of low $\mathrm{H} 3 \mathrm{~K} 9 \mathrm{me} 2$ levels on the $p h o 1^{+}$promoter when repressed ${ }^{28}$, however, the consequence of such $\mathrm{H} 3 \mathrm{~K} 9 \mathrm{me} 2$ remains uncertain given that our analyses show that the expression of $\mathrm{phol}^{+}$or $\operatorname{tgp} 1^{+}$is unaffected by loss of RNAi/ heterochromatin. We note that our findings are in agreement with previous expression profiling analyses, showing unaltered tgp $1^{+}$and $p h o 1^{+}$levels in S. pombe cells lacking RNAi/ heterochromatin ${ }^{40}$. In contrast, transcripts arising from bone fide heterochromatin in centromeric outer repeats are clearly elevated when RNAi/heterochromatin is defective. Thus, our analyses indicate that the repression of both $\operatorname{tgp} 1^{+}$and $p h o 1^{+}$is unlikely to involve regulated heterochromatin in wild-type cells. Instead, we favour a model whereby $\operatorname{tgp} 1^{+}$and $p h o 1^{+}$are repressed by a transcriptional interference mechanism.

Transcriptional interference is well-established in many systems. In the bacterium Escherichia coli, the gene encoding the $c l r$ transcriptional activator is repressed in response to nitrogen starvation by the act of lncRNA transcription from an alternate upstream promoter ${ }^{41}$. In the single celled eukaryote $S$. cerevisiae, which lacks RNAi and heterochromatin, transcription of the SRG1 lncRNA into the SER3 promoter, or heterologous promoters, was found to alter nucleosome density and interfere with transcription factor binding ${ }^{8-10}$. Similarly, in $S$. cerevisiae, non-coding transcription over the IME1 (ref. 20), GAL7 (ref. 42) and FLO11 (ref. 43) promoters prevent gene induction. Analogous mechanisms have also been reported in multicellular eukaryotes. For example, the Drosophila Ubx gene $^{44}$, the human dihydrofolate reductase gene ${ }^{45}$ and the imprinted Igf $2 r$ gene in mammals ${ }^{46}$ are repressed independent of RNAi or transient heterochromatin formation by non-coding transcription into their respective promoters. These examples illustrate that transcriptional interference is a simple, conserved mechanism for modulating specific genes without requiring additional trans-acting regulatory factors. Our results are consistent with both $n c$-tgp1 and $n c$-phol mediating repression of downstream genes $\left(\operatorname{tgp} 1^{+}\right.$and $p h o 1^{+}$, respectively) by transcriptional interference, not by the formation of transient heterochromatin. We base this conclusion on our findings that: (i) $\operatorname{tgp} 1^{+}$and pho $1^{+}$expression is unaffected by loss of RNAi/heterochromatin; (ii) $\mathrm{H} 3 \mathrm{~K} 9 \mathrm{me} 2$ is not associated with $\operatorname{tgp} 1^{+}$or $p h o 1^{+}$loci in wildtype cells; (iii) $n c$-tgp1 transcription declines when $\operatorname{tgp} 1^{+}$is induced $\left(-\mathrm{PO}_{4}\right)$; (iv) loss of the $n c$-tgp1 transcript allows induction of $\operatorname{tgp} 1^{+}$under normally repressive $\left(+\mathrm{PO}_{4}\right)$ conditions (similarly, loss of lncRNA transcription upstream induces phol $^{+}$in repressive medium ${ }^{27,28}$ ); (v) transcription of $n c$-tgp 1 by a thiamine-repressible promoter brings $\operatorname{tg} p 1^{+}$under the control of thiamine, rather than phosphate; (vi) RNAPII and nucleosome density is increased over the $\operatorname{tg} p 1^{+}$promoter region when the repressive $n c$-tgp1 RNA is transcribed and (vii) the Pho7 activator binds the tgp $1^{+}$promoter region when $n c$-tgp1 transcription is lost.

Genome-wide RNA sequencing has allowed the detection of a large number of lncRNAs in a variety of species. However, it remains unclear how many of these lncRNA are functional transcripts that act to influence gene expression and/or chromatin landscapes. Examples such as Xist RNA in mammals and roX RNAs in Drosophila represent functional transcripts that are critical for mediating dosage compensation by altering chromatin status and expression levels from sex chromosomes ${ }^{47}$. However, enthusiasm for lncRNA function has been somewhat dampened by reports showing that the ablation in animal models of some of the best-characterized lncRNAs (for example, HOTAIR, MALAT1, Kcnq1ot1, NEAT1) exhibited less dramatic or undetectable phenotypes ${ }^{48-53}$. Of the discrete stable lncRNAs that we deleted in fission yeast, only one ( $n c R N A .1343)$ had an obvious phenotype in the growth conditions tested. Detailed analysis was required to reveal that deletion of $n c R N A .1343$ actually affected expression of a divergent unstable lncRNA (nc-tgp1) transcribed in the opposite orientation as the annotated locus. Only after further manipulation and analyses could we conclude that the expression of $n c$-tgp1 interferes with the expression of $\operatorname{tgp} 1^{+}$downstream. The fact that the unstable 
$n c$-tgp 1 transcript is the functional partner of the apparently nonfunctional stable $n c-1343$ RNA transcribed from the same bidirectional promoter demonstrates the importance of comprehensive analyses of ncRNAs and the consequences of their deletion. Based on our analyses. we surmise that the low level expression of $n c-1343$ represents transcriptional noise, resulting as a byproduct of ample $n c$-tgp1 transcription. The syntenic conservation of ncRNA.1343 within the Schizosaccharomyces genus ${ }^{31}$ hints at the possibility of a conserved regulatory mechanism that involves lncRNA transcription into the promoter region of $\operatorname{tgp} 1^{+}$in related species. Thus, although genome-wide approaches can rapidly catalogue the presence and response of various lncRNAs to different conditions, much more detailed locus-specific analyses is required to pinpoint the function of each individual lncRNA with respect to cis regulation of nearby genes or trans regulation of genes at distal loci.

\section{Methods}

Yeast strains, plasmids and standard techniques. S. pombe strains used in this study are listed in Supplementary Table 1. Standard methods were used for fission yeast growth, genetics and manipulations ${ }^{54}$. All strains were grown in Yeast extract plus supplement medium (YES), unless otherwise indicated. For phosphate starvation experiments, cells were grown to mid-log phase in YES medium, washed twice in $\mathrm{dH}_{2} \mathrm{O}$, and then grown for indicated times in Pombe minimal glutamate (PMG) synthetic medium without $\mathrm{Na}_{2} \mathrm{HPO}_{4}\left(-\mathrm{PO}_{4}\right)$. Genetic deletions and protein tagging were carried out by lithium acetate transformation. All genetic modifications were confirmed by colony PCR. Plasmids were transformed by electroporation. Selections were performed on PMG/agar plates with according auxotrophy or on YES/agar plates with appropriate antibiotic(s) and grown at $32^{\circ} \mathrm{C}$. Serial (1:4) dilutions of equal number of cells were spotted onto YES/agar and grown at $32^{\circ} \mathrm{C}$, unless indicated otherwise. For drug-sensitivity experiments, cells were spotted onto YES/agar or PMG/agar with DMSO or TBZ $\left(20 \mu \mathrm{g} \mathrm{ml}^{-1}\right)$, $\mathrm{HU}(10 \mathrm{mM})$, caffeine $(15 \mathrm{mM})$ and $\mathrm{H}_{2} \mathrm{O}_{2}(1 \mathrm{mM})$. For ultraviolet-sensitivity experiments, spotted cells were ultraviolet-irradiated at $80 \mathrm{~J} \mathrm{~m}^{-2}$ with a Stratalinker UV Crosslinker and grown in the dark at $25^{\circ} \mathrm{C}$. The plasmids containing lac $Z$ under the control of the $n c$-tgpl and $n c-1343$ bidirectional promoter were cloned as follows. The non-coding promoter was amplified from $S$. pombe genomic DNA in both orientations (using lacZ_1_F/lacZ_1_R and lacZ_2_F/lacZ_2_R primer pairs; see Supplementary Table 2) and ligated into pREP vector containing lac $Z$ using PstI/SalI restriction sites. To test if $n c$-tgp 1 can repress $\operatorname{tgp} 1^{+}$in trans, the $n c$ - $\operatorname{tg} p 1$ transcription unit was amplified from $S$. pombe genomic DNA (using nc-tgp1_SalI_F and nc-tgp1_XmaI_R primer pairs, see Supplementary Table 2) and ligated into pREP3x using SalI/XmaI restriction sites.

Liquid assay for $\boldsymbol{\beta}$-galactosidase activity. Assays for $\beta$-galactosidase activity were performed as described ${ }^{55}$. Briefly, yeast containing vectors expressing lac $Z$ under the control of various promoters were grown to $\log$ phase $\left(\mathrm{OD}_{595}\right.$ of $\left.\sim 0.5\right)$ in selective media. Cells were permeabolized by SDS/chloroform. Cell extracts were equilibrated at $30^{\circ} \mathrm{C}$ for $5 \mathrm{~min}$ before the addition of ortho-Nitrophenyl- $\beta$ galactoside (ONPG). The reaction was stopped with $\mathrm{Na}_{2} \mathrm{CO}_{3}$ once the solution turned yellow and elapsed time was recorded. Cell debris was spun and the $\mathrm{OD}_{420}$ was measured. Units were calculated as follows: Units/OD $=1000 \times\left(\mathrm{OD}_{420} /\right.$ Volume $\times$ Time $\left.\times \mathrm{OD}_{595}\right)$

Chromatin and RIP. Cells were grown to mid-log phase at $32{ }^{\circ} \mathrm{C}$ in YES. For phosphate starvation experiments, cells in mid-log phase were washed twice in $\mathrm{dH}_{2} \mathrm{O}$ before being grown in PMG $\left(-\mathrm{PO}_{4}\right)$ for $4 \mathrm{~h}$. ChIP was performed essentially as described ${ }^{12}$. Briefly, cells were fixed with $1 \%$ paraformaldehyde for $15 \mathrm{~min}$ at room temperature. Cells were lysed by bead beating (Biospec Prodcutes) and sonicated using a Bioruptor (Diagenode) sonicator at $5{ }^{\circ} \mathrm{C}$ on high for a total of 20 min (30 s ON/OFF cycles). Five microlitres of Rpb1 antibody (\#2629; Cell Signaling), $2 \mu \mathrm{l}$ GFP antibody (G10362; Life Technologies), $2 \mu \mathrm{l} \mathrm{H3}$ antibody (ab1791; Abcam) and $1 \mu$ l of H3K9me2 antibody (m5.1.1; ref. 56) were used for IPs. RIP experiments were performed essentially as described ${ }^{13}$. Hisx6-TEV-Protein A-tagged Mmil was captured from cell lysate with IgG Dynabeads (Life Technologies). Mmil-bound RNA was isolated by phenol-chloroform extraction, DNase treated and reverse transcribed. Quantitative analysis was performed by $\mathrm{qPCR}$.

RNA analysis. RNA was isolated from $S$. pombe using RNeasy Mini- or Midi-Kits as per manufacturer's instructions (Qiagen). For RT-qPCR experiments, first strand complementary DNA synthesis was performed on Turbo DNase (Life Technologies) treated RNA using random hexamers and Superscript III (Invitrogen) as per manufacturer's instructions. Negative controls lacking RT were performed alongside all RT-qPCR experiments. Northern analysis of long non-coding transcripts was performed using UTP- $\left[\mathrm{a}^{32} \mathrm{P}\right]$-labelled RNA probes as described ${ }^{57}$ Transcription start sites were mapped using the SMARTer RACE complementary DNA Amplification Kit as per manufacturer's instructions (Clontech).

Quantitative real-time PCR. Primers used in this study are listed in Supplementary Table 2. qPCR was performed using SYBR Green on a Roche Lightcycler. Data was analyzed with LightCycler 480 Software 1.5.0.39. RT-qPCR levels were calculated by normalizing product of interest to an internal reference gene $\left(a c t 1^{+}\right)$. Expression levels were expressed relative to levels detected in wild-type cells. ChIP enrichments were calculated as the ratio of product of interest from IP sample normalized to the corresponding input sample and expressed as '\%IP'. Error bars represent s.e.m., resulting from at least three independent replicates.

\section{References}

1. Guttman et al. Chromatin signature reveals over a thousand highly conserved large non-coding RNAs in mammals. Nature 458, 223-227 (2009).

2. Ponting, C. P., Oliver, P. L. \& Reik, W. Evolution and functions of long noncoding RNAs. Cell 136, 629-641 (2009).

3. Mitchell, P., Petfalski, E., Shevchenko, A., Mann, M. \& Tollervey, D. The exosome: a conserved eukaryotic RNA processing complex containing multiple $3^{\prime} \rightarrow 5^{\prime}$ exoribonucleases. Cell 91, 457-466 (1997).

4. Struhl, K. Transcriptional noise and the fidelity of initiation by RNA polymerase II. Nat. Struct. Mol. Biol. 14, 103-105 (2007).

5. Wilusz, J. E., Sunwoo, H. \& Spector, D. L. Long noncoding RNAs: functional surprises from the RNA world. Genes Dev. 23, 1494-1504 (2009).

6. Li, B., Carey, M. \& Workman, J. L. The role of chromatin during transcription Cell 128, 707-719 (2007).

7. Hirota, K. et al. Stepwise chromatin remodeling by a cascade of transcription intiation of non-coding RNAs. Nature 456, 130-134 (2008).

8. Hainer, S. J., Pruneski, J. A., Michell, R. D., Monteverde, R. M. \& Martens, J. A Intergenic transcription causes repression by directing nucleosome assembly. Genes Dev. 25, 29-40 (2011).

9. Martens, J. A., Laprade, L. \& Winston, F. Inter Thebault genic transcription is required to repress Saccharomyces cerevisiae SER3 gene. Nature 429, 571-574 (2004).

10. Thebault, P. et al. Transcription regulation by the noncoding RNA SRG1 requires Spt2-dependent chromatin deposition in the wake of RNA polymerase II. Mol. Cell Biol. 31, 1288-1300 (2011).

11. Fejes-Toth, K. et al. Post-transcriptional processing generates a diversity of $5^{\prime}$-modified long and short RNAs. Nature 457, 1028-1032 (2009).

12. Bayne, E. H. et al. Stcl: a critical link between RNAi and chromatin modification required for heterochromatin integrity. Cell 140, 666-677 (2010).

13. Motamedi, M. R. et al. Two RNAi complexes, RITS and RDRC, physically interact and localize to noncoding centromeric RNAs. Cell 119, 789-802 (2004).

14. Verdel, A. et al. RNAi-mediated targeting of heterochromatin by the RITS comples. Science 303, 672-676 (2004).

15. Volpe, T. A. et al. Regulation of heterochromatin silencing and histone $\mathrm{H} 3$ lysine-9 methylation by RNAi. Science 297, 1833-1837 (2002).

16. Rinn, J. L. et al. Functional demarcation of active and silent chromatin domains in human HOX loci by non-coding RNAs. Cell 129, 1311-1323 (2007).

17. Ørom, U. A. et al. Long noncoding RNAs with enhancer-like function in human cells. Cell 143, 46-58 (2010).

18. Camblong, J., Iglesias, N., Fickentscher, C., Dieppois, G. \& Stutz, F. Antisense RNA stabilization induces transcriptional gene silencing via histone deacetylation in S. cerevisiae. Cell 131, 706-717 (2007).

19. Houseley, J., Rubbi, L., Grunstein, M., Tollervey, D. \& Vogelauer, M. A ncRNA modulates histone modification and mRNA induction in the yeast GAL gene cluster. Mol. Cell 32, 685-695 (2008).

20. van Werven, F. J. et al. Transcription of two long non-coding RNAs mediates mating-type control of gametogenesis in budding yeast. Cell 150, 1170-1181 (2012).

21. Heo, J. B. \& Sung, S. Vernalization-mediated epigenetic silencing by a long intronic noncoding RNA. Science 331, 76-79 (2011).

22. Wang, K. C. et al. A long noncoding RNA maintains active chromatin to coordinate homeotic gene expression. Nature 472, 120-124 (2011).

23. Hiriart, E. et al. Mmil RNA surveillance machinery directs RNAi complex RITS to specific meiotic genes in fission yeast. EMBO J. 31, 2296-2308 (2012).

24. Yamanaka, S. et al. RNAi triggered by specialized machinery silences developmental genes and retrotransposons. Nature 493, 557-560 (2013).

25. Zofall, M. et al. RNA elimination machinery targeting meiotic mRNAs promotes facultative heterochromatin formation. Science 335, 96-100 (2012).

26. Harigaya, Y. et al. Selective elimination of messenger RNA prevents an incidence of untimely meiosis. Nature 442, 45-50 (2006).

27. Lee, N. N. et al. Mtr4-like protein coordinates nuclear RNA processing for heterochromatin assembly and for telomere maintenance. Cell 155, 1061-1074 (2013). 
28. Shah, S., Wittmann, S., Kilchert, C. \& Vasilieva, L. IncRNA recruits RNAi and the exosome to dynamically regulate phol expression in response to phosphate levels in fission yeast. Genes Dev. 28, 213-244 (2014).

29. Pang, K. C., Frith, M. C. \& Mattick, J. S. Rapid evolution of noncoding RNAs: lack of conservation does not mean lack of function. Trends Genet. 22, 1-5 (2006).

30. Ulitsky, I., Shkumatava, A., Jan, C. H., Sive, H. \& Bartel, D. P. Conserved function of lincRNAs in vertebrate embryonic development despite rapid sequence evolution. Cell 147, 1537-1550 (2011).

31. Rhind, N. et al. Comparative functional genomics of the fission yeasts. Science 332, 930-936 (2011).

32. Wilhelm, B. T. et al. Dynamic repertoire of a eukaryotic transcriptome surveyed at single-nucleotide resolution. Nature 453, 1239-1243 (2008).

33. Leong, H. S. et al. A global non-coding RNA system modulates fission yeast protein levels in response to stress. Nat. Commun. 5, 3947 (2014).

34. Almaguer, C., Mantella, D., Perez, E. \& Patton-Vogt, J. P. Inositol and phosphate regulate GIT1 transcription and glycerophophoinositol incorporation in Saccharomyces cerevisiae. Eukaryot. Cell 2, 729-736 (2003).

35. Carter-O’Connell, I., Peel, M. T., Wykoff, D. D. \& O’Shea, E. Genome-wide characterization of the phosphate starvation response in Schizosaccharomyces pombe. BMC Genomics 13, 697 (2012).

36. Henry, T. C. et al. Systematic screen of Schizosaccharomyces pombe deletion collection uncovers parallel evolution of the phosphate signal transduction pathway in yeasts. Eukaryot. Cell 10, 198-206 (2011).

37. Yuan, G. C. et al. Genome-scale identification of nucleosome positions in S. cerevisiae. Science 309, 626-630 (2005).

38. Bitton, D. A. et al. Programmed fluctuations in sense/antisense transcript ratios drive sexual differentiation in S. pombe. Mol. Syst. Biol. 7, 559 (2011).

39. Reyes-Turcu, F. E., Zhang, K., Zofall, M., Chen, E. \& Grewal, S. I. Defects in RNA quality control factors reveal RNAi-independent nucleation of heterochromatin. Nat. Struct. Mol. Biol. 18, 1132-1138 (2011).

40. Hansen, K. R. et al. Global effects on gene expression in fission yeast by silencing and RNA interference machineries. Mol. Cell Biol. 25, 590-601 (2005).

41. Zafar, M. A., Carabetta, V. J., Mandel, M. J. \& Silhavy, T. J. Transcriptional occlusion caused by overlapping promoters. Proc. Natl Acad. Sci. USA 111, 1557-1561 (2014)

42. Greger, I. H., Aranda, A. \& Proudfoot, N. Balancing transcription interference and initation on the GAL7 promoter of Saccharomyces cerevisiae. Proc. Natl Acad. Sci. USA 97, 8415-8420 (2000).

43. Bumgarner, S. L. et al. Toggle involving cis-interfering noncoding RNAs controls variegated gene expression in yeast. Proc. Natl Acad. Sci. USA 106, 18321-18326 (2009).

44. Petruk, S. et al. Transcriptional elongations of non-coding bxd RNAs promoted by the Trithorax TAC1 complex represses Ubx by a transcriptional interference mechanism. Cell 127, 1209-1221 (2006).

45. Martianov, I., Ramadass, A., Barros, A. S., Chow, N. \& Akoulitchev, A. Repression of the human dihydrofolate reductase gene by a non-coding interfering transcript. Nature 445, 666-670 (2007).

46. Latos, P. A. et al. Airn transcriptional overlap, but not its lncRNA products, induces imprinted Igf2r silencing. Science 338, 1469-1472 (2012).

47. Lee, J. T. \& Bartolomei, M. S. X-activation, imprinting, and long non-coding RNAs in health and disease. Cell 152, 1308-1323 (2013).

48. Eißmann, M. et al. Loss of the abundant nuclear non-coding RNA MALAT1 is compatible with life and development. RNA Biol. 9, 1076-1087 (2012).

49. Korostowski, L., Sedlak, N. \& Engel, N. The Kcnqlot1 long non-coding RNA affects chromatin and expression of Kcnq1, but does not regulate its imprinting in the developing heart. PLOS Genet. 8, e1002956 (2012).
50. Nakagawa, S. et al. Malatl is not an essential component of nuclear speckles in mice. RNA 18, 1487-1499 (2012).

51. Schorderet, P. \& Duboule, D. Structural and functional differences in the long non-coding RNA hotair in mouse and human. PLOS Genet. 7, e1002071 (2011).

52. Zhang, B. et al. The lncRNA Malat1 is dispensable for mouse development but its transcription plays a cis-regulatory role in the adult. Cell Rep. 2, 111-123 (2012).

53. Nakagawa, S., Naganuma, T., Shioi, G. \& Hirose, T. Paraspeckles are subpopulation-specific nuclear bodies that are not essential in mice. J. Cell Biol. 193, 31-39 (2011)

54. Moreno, S., Klar, A. \& Nurse, P. Molecular genetic analysis of fission yeast Schizosaccharomyces Pombe. Methods Enzymol. 194, 795-823 (1991).

55. Guarente, L. Yeast promoters and lacZ fusions designed to study expression of cloned genes in yeast. Methods Enzymol. 101, 181-191 (1983).

56. Nakagawachi, T. et al. Silencing effect of CpG island hypermethylation and histone modifications on O6-methylguanine-DNA methyltransferase (MGMT) gene expression in human caner. Oncogene 22, 8835-8844 (2003).

57. Buscaino, A. et al. Raf1 is a DCAF for the Rik1 DDB1-like protein and has separable roles in siRNA generation and chromatin modification. PLOS Genet. 8, e1002499 (2012).

\section{Acknowledgements}

We would like to thank Sandra Catania, Alison Pidoux, Manu Shukla and Sharon White for their technical expertise and input. We are grateful to Takeshi Urano for the H3K9me2 (5.1.1) antibody, Tomoyasu Sugiyama and Lidia Vasilieva for strains, and Steven West and Sander Granneman for critically evaluating the manuscript. R.A. is supported by the Darwin Trust of Edinburgh. The Centre for Cell Biology is supported by core funding from the Wellcome Trust $(092076 / \mathrm{Z} / 10 / \mathrm{Z})$. P.T. is supported by European Commission Network of Excellence EpiGeneSys (HEALTH-F4-2010-257082) to R.C.A. R.C.A. is a Wellcome Trust Principal Research Fellow and this research was supported by the Wellcome Trust (095021/Z/10/Z).

\section{Authors contributions}

R.A. and R.C.A. conceived and designed the experiments. R.A. performed the experiments. P.T. performed bioinformatics analysis. R.A., P.T. and R.C.A. analyzed the data R.A. and R.C.A. wrote the paper.

\section{Additional information}

Supplementary Information accompanies this paper at http://www.nature.com/ naturecommunications

Competing financial interests: The authors declare no competing financial interests.

Reprints and permission information is available online at http://npg.nature.com/ reprintsandpermissions/

How to cite this article: Ard, R. et al. Long non-coding RNA-mediated transcriptional interference of a permease gene confers drug tolerance in fission yeast. Nat. Commun. 5:5576 doi: 10.1038/ncomms6576 (2014).

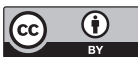

This work is licensed under a Creative Commons Attribution 4.0 International License. The images or other third party material in this article are included in the article's Creative Commons license, unless indicated otherwise in the credit line; if the material is not included under the Creative Commons license, users will need to obtain permission from the license holder to reproduce the material. To view a copy of this license, visit http://creativecommons.org/licenses/by/4.0/ 